\title{
Evolutionary Adaptability of Biological Macromolecules
}

\section{Michael Conrad}

Department of Computer and Communication Sciences, University of Michigan, Ann Arbor, MI 48109, USA

\begin{abstract}
Summary. The degree of gradualism with which tertiary structure and function of protein changes with stepwise changes in primary structure (assumed to be influenced by redundancy of weak bonding) is both a precondition for and consequence of evolution. The resulting selection for degree of gradualism has implications for a number of structural and functional properties of modern proteins as well as for the significance of neutral (so-called non-Darwinian) phenomena in relation to selection.
\end{abstract}

Key words: Molecular evolution - Protein structure and function - Non-Darwinian evolution - Molecular adaptability

Often stepwise changes in the primary structure of proteins are associated with gradual changes in their biological function. Biophysical and evolutionary considerations, taken together, suggest a more general statement about molecular adaptability, viz. that the degree of gradualism is adjustable and adjusted for efficient evolutionary behavior in the course of evolution.

The biophysical consideration is that tertiary structure (which we will take to mean both three-dimensional shape and charge distribution) is generally considered to be decisive for the biological function of proteins (e.g. their catalytic specificity, specificity for self-assembly into larger, quaternary molecular structures). This threedimensional shape arises through a folding process which is determined in the main by weak interactions (e.g. van der Waal's interactions, hydrogen bonds, disulfide bonds, hydrophobic interactions) among the constituent amino acids, so that it is generally stated that tertiary structure is implicit in primary structure. Suppose that amino acids whose main contribution to function is to increase the redundancy of such interactions are added to the protein, i.e. amino acids which overdetermine functionally relevant spatial features of the protein so that these spatial features are implicit in a larger number of variations of the primary structure. Under these circumstances the function of the protein is capable of changing more slowly with changes in primary structure, provided that these changes do not involve amino acids which are particularly critical for function. As pointed out by Zuckerkandl and Pauling (1965) conservatism in 
tertiary structure is also possible because of the structural similarities among different types of amino acids. Thus the degree of gradualism can also be controlled by inserting into the primary structure amino acids with either more or closer structural analogs, in effect utilizing redundancy in features of the shape of different types of amino acids to produce more graded change in the folded shape. In both cases the degree of gradualism with which function changes with primary structure is itself a property implicit in primary structure (since the redundancy of weak interactions or redundancy of amino acid types is a property of primary structure and primary structure determines tertiary structure and therefore function).

The evolutionary argument is that evolution proceeds most rapidly when it proceeds through single genetic changes (cf. Bremermann, 1967; Maynard Smith, 1970) where each genetic type in the sequence has selective value, or is at least viable, and is in general increasingly slow as the number of simultaneous steps required to make the jump from one viable type to another increases. Roughly this is because the probability of such a jump is the product of the probability of each individual change, assuming the individual changes are independent. (A single genetic change could be a point mutation, duplication, recombination or any other indecomposable genetic process. For a sample calculation, taking growth rates of variant types into account, see Conrad, 1972). In terms of proteins this means that the potential rate of evolution is greatest when different useful proteins are derivable from a common ancestor through stepwise changes in primary structure, where each primary structure in the series has functional value. However, there are an enormous number of possible protein sequences (at least on the order of $20^{300}$ ) and it can hardly be expected that the majority of these would have any functional value. This means that the number of functional proteins is extremely sparse in the total number of possible proteins and therefore it is unlikely that primary structures can be selected in such a way that single changes in primary structure produce proteins with very different but nevertheless functional properties. However, according to our considerations in the previous paragraph, they can be selected so that single changes in primary structure are associated with slight changes in function, in which case it is possible for evolution to proceed through the transfer of function mechanism. Moreover, the gradualism type organization is also advantageous from the point of view of reliability, for it means that protein function and therefore the viability of the organism is buffered against slight changes in primary sequence. This becomes more important as the number of different types of proteins which an organism requires increases, for as this number increases it becomes more likely that at least one of these types will be altered by mutation.

The above considerations imply that gradualism is an evolutionary advantage to the species, both in terms of smoothing the adaptive landscape and therefore facilitating evolution and also from the standpoint of increasing reliability and therefore decreasing the proportion of nonfunctional genetic changes. It also implies a possible disadvantage to the individual in terms of energy requirements (since it involves additional weak bonding). However, the disadvantage to the individual increases incrementally with increase in gradualism, while the advantage to the population increases geometrically and more rather than less rapidly as the one step situation is approached (since the emergence time of a new useful protein goes inversely as the product of probabilities 
of the number of required changes). This means that virtually all evolution of new traits must occur in populations which vary in the direction of more nearly optimal gradualism and therefore that selection for practically all protein properties which confer an individual selective advantage will also be tied to selection for the gradualism as well. In this sense the degree of gradualism is both a condition and consequence of evolution by variation and natural selection, from which it follows that species which predominate in the course of evolution are inevitably species whose complement of protein molecules are well adjusted for evolution. This, however, is equivalent to the original statement about molecular adaptability, for it is the same as saying that the degree of gradualism is adjusted for efficient evolutionary behavior in the course of evolution.

The principle of molecular adaptability has a number of implications. These include:

1. Relation of Protein Structure to Mecbanism of Action. The size and structure of proteins is determined in part by amenability to evolution and therefore the structurefunction relation should be interpreted in terms of this amenability as well as in relation to the mechanisms of recognition, catalysis, control, and the processes of structure formation. Much of the structure of any evolved protein is in general superfluous from the purely mechanistic standpoint in the sense that simpler enzymes (less amenable to evolution) might in principle be synthesized which perform the same physiological functions. However, it should be noted that redundancy of weak bonding confers stability on the tertiary and quaternary structures and is also relevant to the uniqueness of the folding process, so that redundancy has a potential physiological as well as evolutionary role. It is the redundancy over and above this minimal redundancy (or redundancy which buffers critical features of the shape) which subserves a specifically evolutionary function.

2. Selective versus Neutral Aspect of Evolution. The rate of evolutionary change of different proteins depends on both the degree of gradualism and on selection pressure. If the degree of gradualism is sufficient, however, certain mutations should be neutral or practically neutral. In this sense the so-called non-Darwinian aspect of evolution (neutral drift of primary sequence, cf. Kimura, 1968; King and Jukes, 1969) is a necessary aspect of Darwinian evolution, for the latter is contingent on adequate gradualism. This means that the paradoxically high genetic variabilities found in nature (cf. Lewontin, 1974) must also be regarded as a necessary aspect, even a precondition for evolution.

3. Isoenzymes. The gradualism in the relation of primary structure to function is useful for interpreting the role of isoenzymes. Not only does this gradualism faciliate evolution and contribute to reliability, but it allows for subtle modulations of function. However, even in the absence of such subtle modulation, the occurrence of isoenzymes would be an inevitable concomitant to gradualism.

4. Relation between Size and Number of Variations in Primary Structure. The variety of primary structures associated with a given enzymatic function should increase with the redundancy implicit in primary structure. This implies greater evolutionary conservatism at the tertiary than at the primary level of structure, known to be the case among the dehydrogenases (Rossman et al., 1975). Increasing redundancy means larger 
proteins or proteins with a larger number of amino acids with close structural analogs, but the converse is not true, except in proteins of a given type. Thus if a given type of enzyme is on the average larger in a particular species, it might be expected that the number of isoenzymes would be greater. Also, those enzymes which have undergone the greatest evolutionary change might be expected to be those with the largest number of amino acids which can be associated with a redundancy function. As the number of sites committed to specific functions increases, a relatively greater amount of redundancy would be required to maintain a comparable potential rate of evolutionary change, and therefore it would be expected that either the potential rate would decrease in this case or the amount of redundancy would increase.

It might be noted that the structural redundancy of certain amino acids, while useable in a variable way to control gradualism, is a fixed feature of present day biological systems. However, this redundancy is itself presumably the product of long (but ancient) evolution. Thus, according to the argument in this paper its presence may be interpreted in terms of its contribution to a menability to evolution, although many other factors must certainly have exerted decisive influences as well.

5. Pbysiological role of gradualism. Any physiological process analogous to natural evolution would depend on molecular adaptability in the same way as natural evolution. This includes selective theories of antibody synthesis (Jerne, 1955) and also recent natural selection models of learning (Conrad, 1974).

6. Gradualism at Higher Levels of Organization. Many biological processes (e.g. cellular self-reproduction, immunity, possibly learning) are amenable to both a molecular biological description in terms of events at the primary level of structure and also a dynamical description in terms of rate constants and concentrations (i.e. in terms of the function implicit in tertiary shape). According to the principle of molecular adaptability, stepwise evolutionary changes at the primary level can always be associated with gradual changes in the rate constants and therefore in the dynamics of the system, where the degree of gradualism is adjustable in the course of evolution, and the range of allowable change in the rate constants depends on the structural stability of the system. Thus the principle of molecular adaptability is important for understanding the gradual relation of function change to structure change at all levels of biological organization.

The above considerations suggest that the principle of molecular adaptability is a potentially useful tool for cross-correlating what might be called primary and tertiary biological models, i.e. descriptions of biological systems in terms of events at the primary level of structure and in terms of the rate constants and concentrations. Each of these types of model deal with different aspects of the same system, but aspects which as a practical matter are not easy to encompass in a single model.

Finally we note that the principle of molecular adaptability is important for appreciating novel aspects of information processing in biological systems (Conrad, 1972). Conventional computer systems are constructed from certain definite types of building blocks. Roughly speaking, all fundamental changes in the information processing functions of such systems are mediated by either reconnecting these building blocks or resetting their states. There is no practical mechanism (at present) for replacing building blocks by others which are slightly different, thereby gradually modifying the dynamics of the system. However, this is practical in biological systems 
(because of self-reproduction and molecular folding), which is why such systems are so amenable to evolution. Thus molecular adaptability plays a fundamental role in relation to the origin and development of information processing.

\section{References}

Bremermann, H.J. (1967). Progr. in Theoret. Biol. 1, 59-77

Conrad, M. (1972), Currents in Mod. Biol. (BioSystems) 5, 1-14

Conrad, M. (1972). The importance of molecular hierarchy in information processing. In: Towards a theoretical biology, vol. 4, C.H. Waddington, ed., p. 222. Edinburgh: Edinburgh University Press

Conrad, M. (1974). Molecular information processing in the central nervous system, Part I: Selection circuits in the brain. In: Physics and mathematics of the nervous system, M. Conrad, W. Güttinger, M. Dal Cin, eds., p. 82. Berlin-Heidelberg-New York: Springer

Jerne, N. (1955). Proc. Natl. Acad. Sci. U.S. 41, 849-857

Kimura, M. (1968). Nature 217, 624

King, J., Jukes, T. (1969). Science 164, no. 3881, 788-798

Lewontin, R. (1974). The genetic basis of evolutionary change, pp. 189-271. New York: Columbia University Press

Maynard Smith, J. (1970). Nature 225, no. 5232, 563-564

Rossman, M.G., Liljas, A., Branden, C., Banaszak, L.J. (1975). Evolutionary and structural relationships among dehydrogenases. In: The enzymes, 3rd ed., vol. 11, P.D. Boyer, ed., p. 61. New York: Academic Press

Zuckerkandl, E., Pauling, L. (1965). Evolutionary divergence and convergence in proteins. In: Evolving genes and proteins, V. Bryson, H.J. Vogel, eds., p. 97. New York: Academic Press 